\title{
Perception des boissons caféinées par les adolescents et les jeunes adultes au Canada
}

\author{
Brittany Cormier, B.A.; Jessica L. Reid, M. Sc.; David Hammond, Ph. D.
}

Diffuser cet article sur Twitter

\section{Résumé}

Cette étude visait à évaluer les perceptions et les attitudes des adolescents et des jeunes adultes à l'égard de la caféine et des boissons énergisantes. Un sondage en ligne a été mené auprès de 2036 participants de 12 à 24 ans afin de savoir quelles étaient leurs connaissances sur la caféine et les boissons énergisantes ainsi que leurs perceptions concernant la caféine et l'innocuité des boissons énergisantes. Peu de répondants $(2,1 \%)$ ont été en mesure de préciser l'apport maximal en caféine recommandé par Santé Canada, bien que la plupart (64,9\%) aient indiqué sans se tromper le nombre maximal de boissons énergisantes pouvant être consommé quotidiennement. Seulement 17,5 \% des répondants ont su repérer, parmi quatre boissons présentées, celle qui contenait le plus de caféine. Dans l'ensemble, les jeunes ont généralement une faible connaissance des quantités de caféine contenues dans les boissons et de l'apport en caféine provenant des boissons caféinées.

Mots-clés : caféine, boissons énergisantes, perceptions, attitudes, adolescents

\section{Introduction}

La caféine est généralement sans danger lorsqu'elle est consommée de façon modérée par des adultes ${ }^{1}$. Cependant, les effets de la consommation de caféine sur la santé des jeunes sont de plus en plus préoccupants. La caféine a été associée à une perturbation de la structure du sommeil, à une consommation accrue de sucre et à des problèmes de santé mentale chez les jeunes. De plus, les effets de la consommation de caféine au cours de la période de développement actif du cerveau soulèvent diverses préoccupations ${ }^{2-6}$.

Selon Santé Canada, les jeunes de plus de 12 ans ne devraient pas consommer plus de 2,5 mg de caféine par kilogramme de leur poids par jour ${ }^{1}$. Les données canadiennes sur la consommation de caféine par les jeunes sont limitées ${ }^{7}$, mais des données américaines de 2009-2010 indiquent que $71 \%$ des répondants de 2 à 19 ans avaient consommé de la caféine au cours d'une journée donnée et qu'au moins 10 \% des consommateurs de caféine de 12 à 19 ans en avaient ingéré plus de 2,45 mg/kg par jour8.

Le marché des boissons caféinées poursuit son expansion grâce à de nouveaux produits comme les boissons énergisantes et les concentrés énergisants, dont plusieurs ciblent les jeunes? ${ }^{9}$. Des travaux antérieurs semblent indiquer que de nombreux jeunes ne connaissent pas la teneur en caféine des produits ni les recommandations au sujet de l'apport quotidien en caféine, confondent souvent les boissons caféinées et les boissons non caféinées (p. ex. les boissons pour sportifs) et ont des perceptions mitigées quant à l'innocuité des boissons énergisantes ${ }^{10-13}$. Notre étude a porté sur la connaissance des recommandations relatives à l'apport quotidien maximal en caféine et à la consommation quotidienne maximale de boissons énergisantes, sur la connaissance des quantités relatives de caféine contenues dans les boissons et sur les perceptions concernant les effets de la caféine et l'innocuité des boissons énergisantes.
Points saillants

- Les jeunes avaient généralement une connaissance faible des recommandations précises sur l'apport en caféine. En effet, la grande majorité des répondants a été incapable d'indiquer quel est l'apport quotidien maximal en caféine recommandé.

- En général, les jeunes connaissaient un peu mieux les ingrédients des boissons énergisantes et les recommandations relatives à la consommation de boissons énergisantes que les recommandations précises sur l'apport en caféine.

- Plus du tiers des jeunes ont déclaré que la caféine pouvait les aider à faire du sport et à perdre du poids et ont indiqué qu'il leur semblait sécuritaire de consommer des boissons énergisantes en faisant de l'activité physique.

\section{Méthodologie}

Les données ont été recueillies au moyen d'un questionnaire en ligne rempli en 2015 par 2036 Canadiens.

Un comité d'éthique de la recherche de l'Université de Waterloo a examiné et approuvé l'étude (Bureau d'éthique de la recherche [BER], n 19401).

\section{Mesures}

La formulation exacte et une description plus détaillée de toutes les mesures sont disponibles ailleurs ${ }^{14}$. 
Caféine : connaissances et perceptions

Les participants devaient indiquer s'ils connaissaient l'apport quotidien maximal en caféine recommandé par Santé Canada pour les personnes de leur âge et, le cas échéant, inscrire le nombre de milligrammes. Ils devaient également repérer, parmi les images de quatre boissons (boisson énergisante Monster, $473 \mathrm{ml}$; café Tim Hortons, grand format; Coca-Cola, $591 \mathrm{ml}$; Gatorade, $591 \mathrm{ml}$ ), celle qui contenait le plus de caféine. De plus, les répondants devaient indiquer dans quelle mesure ils étaient d'accord avec sept affirmations (tableau 1), ce qui a permis d'évaluer leur perception des effets de la caféine. Les réponses ont été codées numériquement de 1 (pas du tout d'accord) à 5 (entièrement d'accord), puis additionnées de façon à obtenir un indice des effets positifs perçus de la caféine.

TABLEAU 1

Effets perçus de la caféine et innocuité perçue des boissons énergisantes chez les adolescents et les jeunes adultes

\begin{tabular}{|c|c|c|c|c|c|c|}
\hline Effets & $\begin{array}{l}\text { Pas du tout } \\
\text { d'accord } \\
\%(n)\end{array}$ & $\begin{array}{l}\text { Pas d'accord } \\
\quad \%(\mathbf{n})\end{array}$ & $\begin{array}{l}\text { Neutre } \\
\%(\mathbf{n})\end{array}$ & $\begin{array}{c}\text { D'accord } \\
\% \text { (n) }\end{array}$ & $\begin{array}{c}\text { Entièrement } \\
\text { d'accord } \\
\%(\mathbf{n})\end{array}$ & $\begin{array}{c}\text { Je ne sais pas } \\
\%(\mathbf{n})\end{array}$ \\
\hline $\begin{array}{l}\text { La caféine me rend anxieux. } \\
(\mathrm{n}=2033)\end{array}$ & $10,6 \%$ (216) & $23,6 \%$ (479) & $26,1 \%(530)$ & $20,4 \%$ (414) & $4,9 \%(100)$ & 14,4 \% (294) \\
\hline $\begin{array}{l}\text { J'aime la façon dont la caféine me fait } \\
\text { me sentir. } \\
(\mathrm{n}=2032)\end{array}$ & 7,3 \% (149) & $12,9 \%(262)$ & $38,7 \%$ (786) & $23,6 \%$ (479) & $5,0 \%(102)$ & $12,5 \%(254)$ \\
\hline $\begin{array}{l}\text { La caféine peut m'aider à étudier. } \\
(\mathrm{n}=2035)\end{array}$ & $8,3 \%(169)$ & $13,7 \%$ (279) & $21,6 \%(440)$ & $37,8 \%$ (769) & $9,2 \%(188)$ & $9,3 \%(190)$ \\
\hline $\begin{array}{l}\text { La caféine m'aide à faire du sport. } \\
(\mathrm{n}=2035)\end{array}$ & $18,7 \%$ (381) & $28,1 \%(571)$ & $25,9 \%(527)$ & $13,6 \%(277)$ & $2,0 \% \quad(41)$ & $11,7 \%$ (238) \\
\hline $\begin{array}{l}\text { La caféine crée une dépendance }{ }^{\mathrm{a}} \text {. } \\
(\mathrm{n}=2035)\end{array}$ & $4,3 \% \quad(88)$ & $8,0 \%(164)$ & $14,7 \%$ (299) & $46,8 \%(952)$ & $19,6 \%$ (399) & 6,6 \% (134) \\
\hline Innocuité & $\begin{array}{l}\text { Absolument pas } \\
\text { sécuritaire } \\
\%(\mathbf{n})\end{array}$ & $\begin{array}{c}\text { Probablement } \\
\text { pas sécuritaire } \\
\%(n)\end{array}$ & $\begin{array}{c}\text { Entre les deux } \\
\% \text { (n) }\end{array}$ & $\begin{array}{l}\text { Probablement } \\
\text { sécuritaire } \\
\%(n)\end{array}$ & $\begin{array}{l}\text { Absolument } \\
\text { sécuritaire } \\
\%(n)\end{array}$ & $\begin{array}{c}\text { Je ne sais pas } \\
\%(\mathbf{n})\end{array}$ \\
\hline $\begin{array}{l}\text { Est-il sécuritaire pour vous de } \\
\text { consommer des boissons énergisantes? } \\
(\mathrm{n}=2035)\end{array}$ & $20,6 \%$ & $35,6 \%(725)$ & $24,1 \%(490)$ & $14,9 \%$ (304) & $1,5 \%$ & $3,4 \% \quad(68)$ \\
\hline $\begin{array}{l}\text { Est-il sécuritaire pour les enfants de } \\
\text { consommer des boissons énergisantes? } \\
(\mathrm{n}=2035)\end{array}$ & $66,2 \%(1347)$ & $23,3 \%(475)$ & $5,0 \%(102)$ & $2,5 \% \quad(52)$ & $0,6 \%$ & $2,3 \% \quad(47)$ \\
\hline $\begin{array}{l}\text { Est-il sécuritaire de consommer des } \\
\text { boissons énergisantes pour aider à } \\
\text { étudier? } \\
(\mathrm{n}=2035)\end{array}$ & $17,4 \%$ & $27,5 \%$ (559) & $29,0 \%(590)$ & $16,6 \%$ (338) & $3,1 \% \quad(64)$ & $6,4 \%(131)$ \\
\hline $\begin{array}{l}\text { Est-il sécuritaire pour les personnes de } \\
\text { votre âge de boire plus que le nombre } \\
\text { maximal de canettes/bouteilles par } \\
\text { jour indiqué sur le contenant? } \\
(\mathrm{n}=2034)\end{array}$ & $54,0 \%$ (1 098) & $31,1 \%(632)$ & $7,1 \%(144)$ & $3,7 \% \quad(75)$ & $1,0 \% \quad(19)$ & $3,2 \% \quad(66)$ \\
\hline
\end{tabular}

${ }^{a}$ Cette affirmation fait partie de l'indice de l'innocuité. 


\section{Boissons énergisantes : connaissances et perceptions}

Après avoir vu une image d'une canette de boisson énergisante $(473 \mathrm{ml})$, les participants devaient répondre à la question suivante : "Quel est le nombre maximum de canettes de ce produit que quelqu'un de votre âge devrait consommer en une journée? » Ils devaient également nommer les "principaux ingrédients dans les boissons énergisantes qui donnent le boost ou l'augmentation d'énergie » et préciser si les " principaux ingrédients » des boissons pour sportifs sont les mêmes que ceux qui procurent un regain d'énergie dans les boissons énergisantes. De plus, les répondants devaient indiquer si les boissons énergisantes étaient, à leur avis, bonnes ou mauvaises pour la santé. Finalement, huit énoncés ont permis d'évaluer les perceptions concernant le pouvoir addictif de la caféine et l'innocuité des boissons énergisantes (tableau 1). Les réponses ont été codées numériquement de 1 (absolument pas sécuritaire) à 5 (absolument sécuritaire), puis additionnées de façon à obtenir un indice de l'innocuité perçue des boissons énergisantes. Tant pour l'indice des effets que pour l'indice de l'innocuité, les réponses "Je ne sais pas » ont été recodées comme « Neutre » afin d'éviter qu'elles soient exclues de l'indice. Les réponses manquantes ont quant à elles été exclues. Les réponses aux énoncés ont fait l'objet d'un codage inversé lorsque cela était pertinent.

\section{Analyse des données}

Des analyses statistiques descriptives ont été effectuées, ainsi que des analyses de régression linéaire à l'aide de modèles par paire sélectionnés permettant d'examiner les corrélats sociodémographiques de l'indice des effets perçus de la caféine et de l'indice de l'innocuité perçue des boissons énergisantes $\left[\hat{\mathrm{y}}=\mathrm{B}_{0}+\operatorname{genre}(\mathrm{x})+\operatorname{âge}(\mathrm{x})+\right.$ langue $(\mathrm{x})+$ région $(\mathrm{x})+$ origine ethnique $(\mathrm{x})$ $+\Sigma]$. La correction de Bonferroni a été appliquée pour ajuster tous les seuils $p$ afin de tenir compte des multiples comparaisons par paires. Les pondérations par poststratification ont été établies et appliquées à l'ensemble de données. À moins d'indication contraire, les estimations présentées dans le texte sont pondérées. Toutes les analyses ont été effectuées à l'aide du logiciel IBM SPSS Statistics 24.

\section{Résultats}

\section{Caractéristiques de l'échantillon}

Les caractéristiques démographiques de l'échantillon sont présentées ailleurs ${ }^{14}$. Les répondants étaient âgés de 12 à 24 ans, résidaient au Canada (à l'exclusion des territoires) et étaient majoritairement Blancs (67,0 \%, non pondéré) et anglophones (78,1 \%, non pondéré).

\section{Connaissances sur la caféine}

Dans l'ensemble, 5,1 \% des répondants ( $n=104$ ) ont déclaré connaître l'apport quotidien maximal en caféine recommandé par Santé Canada pour les personnes de leur âge, tandis que $94,9 \%$ ( $n=1928)$ ont répondu "Non » ou "Je ne sais pas ». Toutefois, ceux qui ont déclaré connaître cet apport recommandé n'ont pas tous réussi par la suite à le quantifier correctement; seulement $2,1 \%(n=42)$ des 2036 répondants ont su le faire. Lorsqu'il leur a été demandé de déterminer laquelle des quatre boissons présentées contenait le plus de caféine, la plupart des répondants ont choisi la boisson énergisante $(60,5 \%$, $\mathrm{n}=1231$ ), alors que seulement $17,5 \%$ ( $\mathrm{n}=355$ ) ont répondu sans se tromper que le café était la boisson contenant le plus de caféine. Un pourcentage plus faible de répondants a choisi le Coca-Cola (13,9\%; $\mathrm{n}=283)$ ou le Gatorade $(0,8 \% ; \mathrm{n}=17)$, tandis que $7,3 \%(n=147)$ ont répondu « Je ne sais pas ».

\section{Effets de la caféine}

Comme le tableau 1 l'illustre, la majorité des participants a répondu " Neutre » aux affirmations " La caféine me rend anxieux » et « J'aime la façon dont la caféine me fait me sentir ». La plupart des répondants étaient d'accord avec les affirmations selon lesquelles la caféine pouvait les aider à étudier et les empêchait de bien dormir la nuit, alors qu'ils n'étaient pas d'accord avec les affirmations selon lesquelles la caféine pouvait les aider à perdre du poids ou à faire du sport. La majorité des répondants estimait que la caféine crée une dépendance.

Le tableau 2 montre les résultats des différents modèles de régression linéaire portant sur les corrélats sociodémographiques de l'indice des effets perçus de la caféine et de l'indice de l'innocuité perçue des boissons énergisantes. Le sexe et l'âge sont corrélés de façon significative avec l'indice des effets perçus de la caféine. En effet, les répondants de sexe masculin et les répondants plus âgés ont obtenu des scores plus élevés, ce qui indique que les effets de la caféine sont perçus par ces groupes de façon plus positive.

\section{Connaissances sur les boissons énergisantes}

Après avoir vu l'image d'une canette de boisson énergisante, les répondants étaient priés d'indiquer le nombre maximal de canettes de ce produit qu'une personne de leur âge devrait consommer en une journée. La plupart des répondants $(64,9 \%$, $\mathrm{n}=1$ 318) ont donné la bonne réponse, soit " une » ou moins, 18,7 \% ( $=380)$ ont fourni une réponse erronée $(>1)$ et $16,3 \%(\mathrm{n}=331)$ ont répondu ne pas savoir.

Lorsque les répondants ont été invités à nommer les principaux ingrédients procurant un regain d'énergie dans les boissons énergisantes, l'ingrédient qui a été le plus souvent mentionné a été la caféine $(41,1 \%, \mathrm{n}=836)$, suivie du sucre $(12,8 \%$, $\mathrm{n}=260)$, de la taurine $(10,6 \%, \mathrm{n}=216)$, du guarana $(3,5 \%, \mathrm{n}=70)$, des vitamines $(3,3 \%, \mathrm{n}=66)$, du ginseng $(3,1 \%, \mathrm{n}=64)$ et d'autres ingrédients $(1,1 \%, \mathrm{n}=22)$. La plupart des répondants $(63,2 \%, \mathrm{n}=1286)$ croyaient à raison que les principaux ingrédients des boissons pour sportifs n'étaient pas les mêmes que ceux qui procurent un regain d'énergie dans les boissons énergisantes, mais $7,5 \%(\mathrm{n}=153)$ croyaient qu'il s'agissait des mêmes ingrédients et un pourcentage important $(29,3 \%, \mathrm{n}=596)$ a répondu ne pas savoir.

\section{Perceptions à l'égard des boissons énergisantes}

Les trois quarts des répondants pensaient que les boissons énergisantes étaient mauvaises pour la santé : 46,6\% (n = 950) ont répondu "Mauvaises pour la santé » et 29,6\% ( $\mathrm{n}=603)$ ont répondu « Très mauvaises pour la santé ». Un petit nombre a indiqué "Bonnes pour la santé » $(2,4 \%$, $\mathrm{n}=49)$ ou " Très bonnes pour la santé » $(0,5 \%, \mathrm{n}=11)$, tandis que $17,9 \%$ ( $\mathrm{n}=364$ ) ont choisi « Ni bonnes ni mauvaises pour la santé » et 2,9\% (n=60) ont répondu ne pas savoir.

\section{Perceptions concernant l'innocuité des boissons énergisantes}

Le tableau 1 présente les réponses aux énoncés relatifs à l'innocuité des boissons énergisantes. La plupart des répondants ont indiqué qu’il n'était « Absolument pas sécuritaire » ou « Probablement pas sécuritaire » pour eux de consommer des boissons énergisantes ou encore de consommer des boissons énergisantes en s'entraînant 
TABLEAU 2

Estimations à partir des modèles de régression linéaire pour l'indice des effets perçus de la caféine et l'indice de l'innocuité perçue des boissons énergisantes

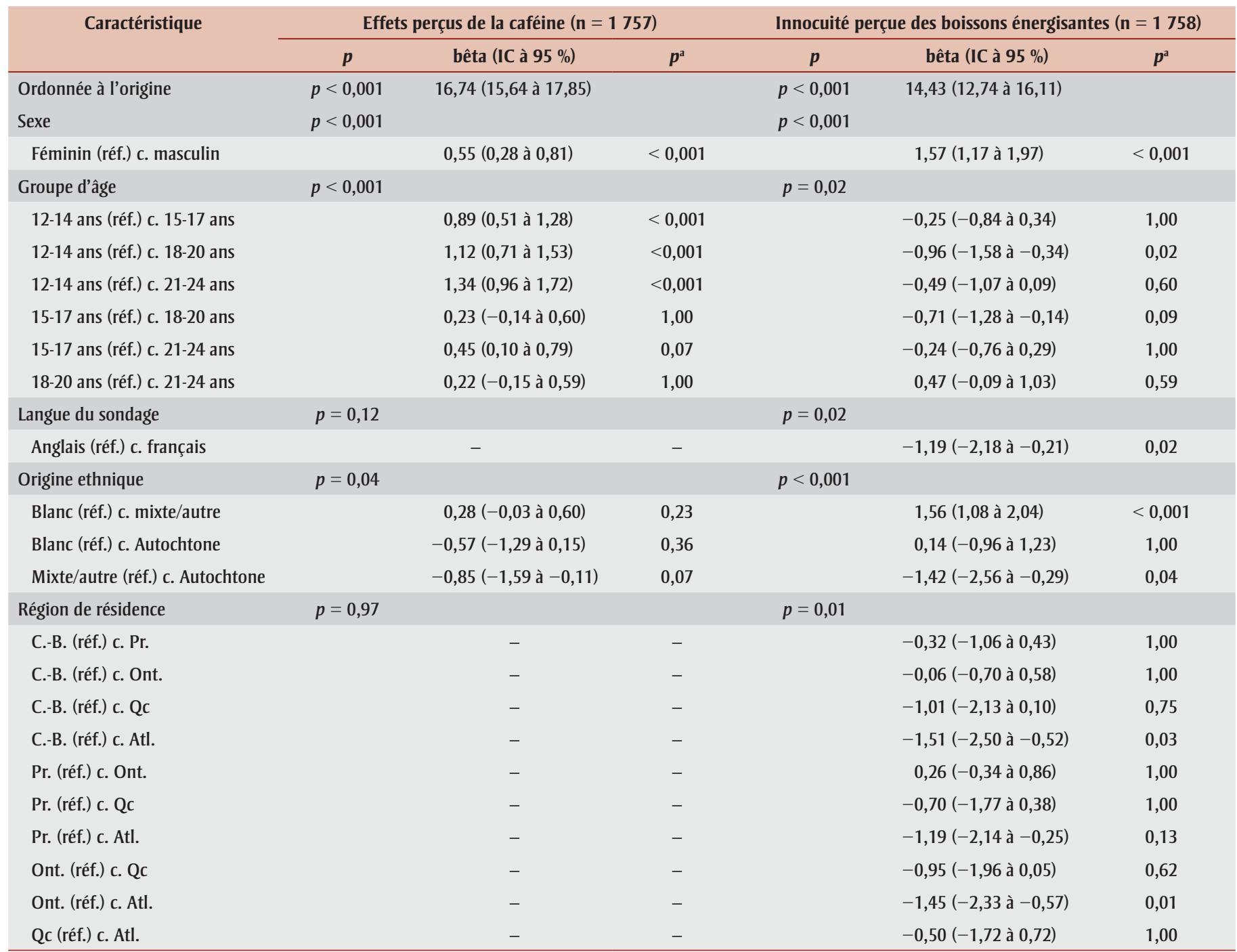

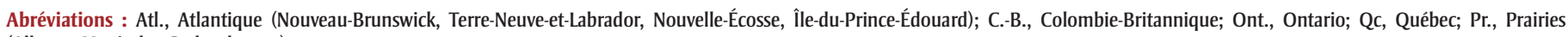
(Alberta, Manitoba, Saskatchewan).

${ }^{a}$ Toutes les valeurs $p$ ont été ajustées à l'aide de la correction de Bonferroni.

- : Sans objet.

ou en faisant du sport. La plupart des répondants ont aussi indiqué qu'il n'était "Absolument pas sécuritaire » pour les enfants et pour les femmes enceintes ou qui allaitent de consommer des boissons énergisantes et qu'il n'était « Absolument pas sécuritaire » de mélanger alcool et boissons énergisantes ou de boire plus que le nombre maximal de canettes ou de bouteilles par jour indiqué sur le contenant.

Le sexe, l'âge, la langue, l'origine ethnique et la région sont tous corrélés de façon significative avec l'indice de l'innocuité perçue des boissons énergisantes (tableau 2). En effet, les répondants de sexe masculin, les répondants de 12 à 14 ans (par rapport à ceux de 18 à 20 ans), les répondants anglophones et les répondants de race ou d'origine ethnique mixte ou autre ont obtenu des scores plus élevés, ce qui indique que les boissons énergisantes sont perçues par ces groupes comme étant plus sécuritaires. Les répondants de la région de l'Atlantique ont obtenu des scores plus faibles, ce qui indique que les boissons énergisantes sont perçues par ces groupes comme étant moins sécuritaires.

\section{Analyse}

Les jeunes avaient généralement une faible connaissance des recommandations précises sur l'apport en caféine. En effet, la plupart des répondants ont été incapables d'indiquer quel est l'apport quotidien maximal recommandé. Bien que Santé Canada ait établi des recommandations ${ }^{1}$, peu de mesures ont été prises pour communiquer ces directives aux consommateurs. De plus, les recommandations sont présentées en $\mathrm{mg} / \mathrm{kg}$ de poids pour les adolescents, ce qui peut s'avérer trop complexe et causer des malentendus.

Les jeunes connaissaient un peu mieux les ingrédients des boissons énergisantes et les recommandations relatives à la consommation de boissons énergisantes. Santé Canada exige que figure sur l'emballage 
des boissons énergisantes une mise en garde relative au nombre maximal de contenants à consommer ${ }^{15}$, une unité de mesure facile à comprendre. Cette exigence pourrait contribuer à expliquer l'écart observé entre les connaissances sur la caféine en général et celles sur les boissons énergisantes en particulier. Même si les répondants connaissaient mieux la consommation maximale recommandée pour les boissons énergisantes que pour la caféine, le tiers d'entre eux a déclaré ne pas connaître la consommation maximale de boissons énergisantes recommandée ou a répondu incorrectement à la question en indiquant un nombre erroné.

Finalement, l'étude a révélé que plus du tiers des jeunes ont déclaré que la caféine pouvait les aider à faire du sport ou à perdre du poids, et qu'il leur semblait sécuritaire de consommer des boissons énergisantes en faisant de l'activité physique. Ce constat est particulièrement préoccupant, car la consommation de caféine durant la pratique d'une activité physique intense n'est pas recommandée par Santé Canada en raison d'un risque accru d'effets indésirables ${ }^{15}$.

\section{Limites}

L'étude comporte des limites : l'existence d'un biais dans les réponses lié, notamment, à la désirabilité sociale et une absence de méthode de recrutement par échantillonnage probabiliste. Toutefois, des pondérations par poststratification ont été utilisées afin d'assurer une distribution représentative des variables démographiques.

\section{Conclusion}

Nos observations sur les jeunes Canadiens laissent penser, d'une part, que les jeunes de sexe masculin perçoivent les effets de la caféine de façon plus positive et considèrent les boissons énergisantes comme étant plus sécuritaires et, d'autre part, que les adolescents plus jeunes ainsi que les jeunes d'origine ethnique mixte ou autre considèrent les boissons énergisantes comme étant plus sécuritaires. Les recherches futures devraient porter sur des méthodes permettant d'améliorer les connaissances des jeunes sur les recommandations et les risques liés à la caféine et aux boissons énergisantes, en particulier pour les groupes qui considèrent les boissons énergisantes comme étant sécuritaires. Compte tenu des tendances du marché, d'autres études devraient également s'intéresser à la consommation de produits caféinés durant l'activité physique et surveiller l'apport en caféine chez les jeunes.

\section{Remerciements}

Cette étude a été financée par une subvention de fonctionnement des Instituts de recherche en santé du Canada (IRSC) (Evaluating the impact of Canada's caffeinated ED policy among youth and young adults), une bourse de nouveau chercheur des IRSC (à DH), une bourse de recherche pour chercheur débutant de l'Institut de recherche de la Société canadienne du cancer (à $\mathrm{DH}$ ) et une chaire en santé publique appliquée des IRSC et de l'ASPC (à DH).

\section{Conflits d'intérêts}

Les auteurs déclarent n'avoir aucun conflit d'intérêts.

\section{Contributions des auteurs et avis}

DH et JLR ont participé à la conception de l'étude et à la collecte de données. Tous les auteurs (BC, JLR et $\mathrm{DH}$ ) ont participé à l'analyse des données et à la préparation du manuscrit, la rédaction et la révision.

Le contenu de l'article et les points de vue qui y sont exprimés n'engagent que les auteurs et ne correspondent pas nécessairement à ceux du gouvernement du Canada.

\section{Références}

1. Santé Canada. La caféine dans les aliments. Ottawa (Ont.) : Santé Canada; 2012. En ligne à : https://www.canada .ca/fr/sante-canada/services/aliments -nutrition/salubrite-aliments/additifs -alimentaires/cafeine-aliments/aliments .html

2. Aepli A, Kurth S, Tesler N, et al. Caffeine consuming children and adolescents show altered sleep behavior and deep sleep. Brain Sciences. 2015; 5:441-455.

3. Keast RS, Swinburn BA, Sayompark D, et al. Caffeine increases sugar-sweetened beverage consumption in a freeliving population: a randomised controlled trial. British Journal of Nutrition. 2015;113:366-371.
4. Richards G, Smith A. Caffeine consumption and self-assessed stress, anxiety, and depression in secondary school children. Journal of Psychopharmacology. 2015;29:1236-1247.

5. Knight CA, Knight I, Mitchell DC, et al. Beverage caffeine intake in US consumers and subpopulations of interest: estimates from the Share of Intake Panel survey. Food and Chemical Toxicology. 2004;42:1923-1930.

6. Arain $\mathrm{M}$, Haque $\mathrm{M}$, Johal $\mathrm{L}$, et al. Maturation of the adolescent brain. Neuropsychiatr Dis Treat. 2013;9: 449-461.

7. Chou T. Wake up and smell the coffee. Caffeine, coffee, and the medical consequences. Western Journal of Medicine. 1992;157:544.

8. Ahluwalia N, Herrick K, Moshfegh A, et al. Caffeine intake in children in the United States and 10-y trends: 20012010. Am J Clin Nutr. 2014;100: 1124-1132.

9. Heckman MA, Sherry K, Mejia D, et al. Energy drinks: an assessment of their market size, consumer demographics, ingredient profile, functionality, and regulations in the United States. Compr Rev Food Sci Food Saf. 2010;9:303-317.

10. Turton P, Piché L, Battram DS. Adolescent attitudes and beliefs regarding caffeine and the consumption of caffeinated beverages. J Nutr Educ Behav. 2016;48:181-189.

11. Thakre TP, Deoras K, Griffin C. Caffeine awareness in children: insights from a pilot study. J Clin Sleep Med. 2015;11:741-746.

12. Costa BM, Hayley A, Miller P. Young adolescents' perceptions, patterns, and contexts of energy drink use. A focus group study. Appetite. 2014;80: 183-189.

13. Bunting $\mathrm{H}$, Baggett A, Grigor J. Adolescent and young adult perceptions of caffeinated energy drinks. A qualitative approach. Appetite. 2013; 65:132-138. 
14. Reid JL, Hammond D. Technical Report: Evaluating the impact of Canada's caffeinated energy drink policy among youth and young adults: Online survey - Wave 2. Waterloo (Ont.) : University of Waterloo; 2016. En ligne à : http://davidhammond.ca /wp-content/uploads/2014/12/2015 -CED-Technical-Report-Final.pdf

15. Santé Canada. Document d'orientation particulier à une catégorie - Autorisation de mise en marché temporaire Boissons énergisantes contenant de la caféine, Ottawa (Ont.) : Santé Canada; 2013. En ligne à : https://www.canada .ca/fr/sante-canada/services/aliments -nutrition/legislation-lignes-directrices /document-reference/document -orientation-categorie-autorisation -mise-marche-temporaire-boissons -energisantes-cafeine.html 